\title{
EXISTENCE OF NONTRIVIAL SOLUTIONS FOR QUASI-LINEAR RESONANT PROBLEMS
}

\author{
AbDesslem AYOUJIL ${ }^{1}$ and AbDel RaChid El AMROUSS ${ }^{1}$
}

\begin{abstract}
Combining the minimax arguments and the Morse Theory, by computing the critical groups at zero, we establish the existence of a nontrivial solution for a class of Dirichlet boundary value problems, with resonance at infinity and zero.
\end{abstract}

Résumé. Par un procédé de minimax et application de la Théorie de Morse, en calculant les groupes critiques en zéro, nous établissons l'existence d'une solution non triviale pour une classe de problèmes de Dirichlet, avec résonance à l'infini et en zéro..

\section{INTRODUCTION}

This paper is mainly concerned to study some classes of resonant elliptic equations. More specifically, we deal with the problem

$$
(\mathcal{P})\left\{\begin{aligned}
-\Delta_{p} u & =f(x, u), & & \text { in } \Omega, \\
u & =0, & & \text { on } \partial \Omega,
\end{aligned}\right.
$$

where $\Omega \subset \mathbb{R}^{N}$ is a bounded domain with smooth boundary $\partial \Omega$ and $\Delta_{p} u:=\operatorname{div}\left(|\nabla u|^{p-2} \nabla u\right), 1<p<\infty$, is the p-Laplacian. We assume that $f: \Omega \times \mathbb{R} \rightarrow \mathbb{R}$ is a Carathéodory function with subcritical growth, that is,

$$
|f(x, t)| \leq c\left(1+|t|^{q-1}\right), \quad \forall t \in \mathbb{R}, \quad \text { a.e } \mathrm{x} \in \Omega
$$

for some $c>0$, and $1 \leq q<p^{*}$ where $p^{*}=\frac{N p}{N-p}$ if $1<p<N$ and $p^{*}=+\infty$ if $N \leq p$.

The growing attention in the study of the p-Laplace operator is widely motivated by the fact that it aries in various applications, e.g. non-Newtonian fluids, reaction-diffusion problems, flow through porus media, nonlinear elasticity, theory of superconductors, petroleum extraction, glacial sliding, astronomy, biology etc...

The problem $(\mathcal{P})$ at resonance with $p \neq 2$ has been studied by few authors. Via directly variational methods or the minimax method, such that as the well-known saddle point theorem or the mountain pass theorem, solvability results for one solution were obtained, we refer to $[6,9,11,15]$ and references therein.

Morse Theory, developed by Chang (cf. [7]) or Mawhin and willem (cf. [13]), is widely used in the study of the existence and multiplicity of solutions of certain nonlinear differential equations arising in the calculus of variations see for example [12]. Thus the computation of critical groups may yield the existence of nontrivial solution to the problem $(\mathcal{P})$.

\footnotetext{
1 Department of Mathematics, Faculty of Sciences, University Mohammed I, Oujda, Morocco ;

e-mail: abayoujil@yahoo.fr \& amrouss@sciences.univ-oujda.ac.ma
} 
From a variational stand point, finding weak solutions of $(\mathcal{P})$ in $W_{0}^{1, p}(\Omega)$ is equivalent to finding critical points of the functional $\Phi$, associated to $(\mathcal{P})$, given by

$$
\Phi(u)=\frac{1}{p} \int_{\Omega}|\nabla u|^{p} d x-\int_{\Omega} F(x, u) d x, \quad u \in W_{0}^{1, p}(\Omega),
$$

where $F(x, t)=\int_{0}^{t} f(x, s) d s$ and $W_{0}^{1, p}(\Omega)$ is the Sobolev space endowed with the norm

$$
\|u\|=\left(\int_{\Omega}|\nabla u|^{p}\right)^{\frac{1}{p}}
$$

It is well known that under the condition $\left(f_{0}\right), \Phi$ is well defined and is a $C^{1}$ functional with its derivative given by

$$
\left\langle\Phi^{\prime}(u), v\right\rangle=\int_{\Omega}|\nabla u|^{p-2} \nabla u \nabla v d x-\int_{\Omega} f(x, u) v d x, \quad \forall u, v \in W_{0}^{1, p}(\Omega),
$$

where $\langle.,$.$\rangle is the duality pairing between W_{0}^{1, p}(\Omega)$ and $W^{-1, p^{\prime}}(\Omega)$.

Obviously, if $f(x, 0) \equiv 0$, then the problem $(\mathcal{P})$ possesses the trivial solution $u \equiv 0$. In this case, the purpose of the present paper is to assure the existence of nontrivial solutions for $(\mathcal{P})$. With this aim, we need to introduce a conditions that give us information about the behaviors of the perturbed function $f(x, t)$ or its primitive $F(x, t)$ near infinity and near zero.

Let $\lambda_{1}$ and $\lambda_{2}$ be the first and the second eigenvalues of the p-homogeneous boundary problem

$$
\left\{\begin{aligned}
-\Delta_{p} u & =\lambda|u|^{p-2} u, & & \text { in } \Omega, \\
u & =0, & & \text { on } \partial \Omega .
\end{aligned}\right.
$$

It is well known, that $\lambda_{1}>0$ is simple and isolated point in the spectrum $\sigma\left(-\Delta_{p}\right)$ of $-\Delta_{p}$ on $W_{0}^{1, p}(\Omega)(\mathrm{cf}$. [2]), which has an associated eigenfunction $\varphi_{1}>0$.

Now we state the assumptions and the main result.

$\left.\left(f_{1}\right) \quad \lim _{|t| \rightarrow \infty}[t f(x, t)-p F(x, t))\right]=-\infty$ uniformly for a.e $x \in \Omega$.

$\left(f_{2}\right) \quad \lambda_{1} \leq \liminf _{|t| \rightarrow \infty} \frac{p F(x, t)}{|t|^{p}} \leq \limsup _{|t| \rightarrow \infty} \frac{p F(x, t)}{|t|^{p}}:=k(x)<\lambda_{2}$ uniformly a.e $x \in \Omega$.

$\left(f_{3}\right) \quad$ There exist $\mu \in(0, p)$ and $\delta>0$ such that

$$
0<\mu F(x, t) \leq t f(x, t), \text { for a.e. } x \in \Omega, 0<|t| \leq \delta,
$$

and

$$
\liminf _{|t| \rightarrow 0} \frac{\mu F(x, t)-t f(x, t)}{|t|^{p}} \geq \alpha>\lambda_{1}\left(\frac{\mu}{p}-1\right) \text { uniformly a.e } x \in \Omega,
$$

with $\alpha$ be a constant non positive.

Theorem 0.1. Assume $\left(f_{0}\right)-\left(f_{3}\right)$. Then the problem $(\mathcal{P})$ has at least one nontrivial solution.

Before to presenting the proof, we need to collect some concepts and results that be used below. 


\section{Preliminary Results}

Let $\mathrm{X}$ be a Banach space, given a $\Phi \in C^{1}(X, \mathbb{R})$ and $\gamma, c \in \mathbb{R}$, we set

$$
\left\{\begin{array}{l}
\Phi^{\gamma}=\{u \in X: \Phi(u) \leq \gamma\} \\
K=\left\{u \in X: \Phi^{\prime}(u)=0\right\} \\
K_{c}=\left\{u \in X: \Phi(u)=c, \Phi^{\prime}(u)=0\right\}
\end{array}\right.
$$

Throughout this paper, we use a generalization of the classical Plais-Smale $(P S)$ condition which has been introduced by Cerami (cf. [4]).

Definition 1.1. Given $c \in \mathbb{R}$, we say that $\Phi \in C^{1}(X, \mathbb{R})$ satisfies condition $\left(C_{c}\right)$, if

$\left.c_{i}\right)$ any bounded sequence $\left(u_{n}\right) \subset X$ such that $\Phi\left(u_{n}\right) \rightarrow c$ and $\Phi^{\prime}\left(u_{n}\right) \rightarrow 0$ has a convergent subsequence, $\left.c_{i i}\right)$ there is constants $\delta, R, \alpha>0$ such that

$$
\left\|\Phi^{\prime}(u)\right\|_{X^{\prime}}\|u\|_{X} \geq \alpha, \quad \forall u \in \Phi^{-1}([c-\delta, c+\delta]) \text { with }\|u\|_{X} \geq R
$$

If $\Phi$ satisfies condition $\left(C_{c}\right)$ for every $c \in \mathbb{R}$, we simply say that $\Phi$ satisfies $(C)$.

Now, recall the notion of critical groups at an isolated critical point, we refer the readers to [7,13] for more information.

Definition 1.2. Suppose $u \in K$ is an isolated critical point of a functional $\Phi \in C^{1}(X, \mathbb{R})$, with $\Phi(u)=c$. We define the $q^{\text {th }}$ critical group of $\Phi$ at $u$ with integer coefficients $\mathbb{Z}$ by

$$
C_{q}(\Phi, u)=H_{q}\left(\Phi^{c} \cap U, \Phi^{c} \cap U \backslash\{u\}\right), \quad q \in \mathbb{Z},
$$

where $U$ is a neighborhood of $u$, and $H_{q}(A, B)$ is the q-th homology group of the topological pair $(A, B)$ over the $\operatorname{ring} \mathbb{Z}$.

We have the following Morse relation between the critical groups and homological characterization of sublevel sets (cf. $[8,16])$.

Theorem 1.1. Suppose $\Phi \in C^{1}(X, \mathbb{R})$ and satisfies $(C)$ condition. If $c \in \mathbb{R}$ is an isolated critical value of $\Phi$, with $K_{c}=\left\{u_{j}\right\}_{j=1}^{n}$, then, for every $\varepsilon>0$ sufficiently small, we have

$$
H_{q}\left(\Phi^{c+\epsilon}, \Phi^{c-\epsilon}\right)=\oplus_{1 \leq j \leq n} C_{q}\left(\Phi, u_{j}\right)
$$

Remark 1.1. From theorem 1.1 follows that if $H_{q}\left(\Phi^{c+\epsilon}, \Phi^{c-\epsilon}\right)$ is nontrivial for some $q$, then there exists a critical point $u \in K_{c}$ with $C_{q}(\Phi, u) \nsubseteq 0$. Furthermore, when $C_{q}(\Phi, 0) \cong 0$ for all $q$, we get that $u \neq 0$.

We will use the following theorem, which is proved with (PS) condition see for example [13].

Theorem 1.2. Assume that $\Phi \in C^{1}(X, \mathbb{R})$, there exists $u_{0}, u_{1} \in X$ and a bounded open neighborhood $\Omega$ of $u_{0}$ such that $u_{1} \in X \backslash \bar{\Omega}$ and

$$
\inf _{\partial \Omega} \Phi>\max \left(\Phi\left(u_{0}\right), \Phi\left(u_{1}\right)\right) .
$$

Let $\Gamma=\left\{g \in C([0,1], X): g(0)=u_{0}, g(1)=u_{1}\right\}$ and

$$
c=\inf _{g \in \Gamma} \max _{s \in[0,1]} \Phi(g(s)) .
$$

If $\Phi$ satisfies the $(C)$ condition over $X$ and if each critical point of $\Phi$ in $K_{c}$ is isolated in $X$, then there exists $u \in K_{c}$ such that

$$
\operatorname{dim} C_{1}(\Phi, u) \geq 1
$$


Proof. Let $\varepsilon>0$ be such that $c-\varepsilon>\max \left(\Phi\left(u_{0}\right), \Phi\left(u_{1}\right)\right)$ and $\mathrm{c}$ is the only critical value of $\Phi$ in $[c-\varepsilon, c+\varepsilon]$. Consider the exact sequence

$$
\ldots \rightarrow H_{1}\left(\Phi^{c+\epsilon}, \Phi^{c-\epsilon}\right) \stackrel{\partial}{\rightarrow} H_{0}\left(\Phi^{c-\epsilon}, \emptyset\right) \stackrel{i_{*}}{\rightarrow} H_{0}\left(\Phi^{c+\epsilon}, \emptyset\right) \rightarrow \ldots
$$

where $\partial$ is the boundary homomorphism and $i_{*}$ is induced by the inclusion mapping $i:\left(\Phi^{c-\epsilon}, \emptyset\right) \rightarrow\left(\Phi^{c+\epsilon}, \emptyset\right)$. The definition of $\mathrm{c}$ implies that $u_{0}$ and $u_{1}$ are path connected in $\Phi^{c+\varepsilon}$ but not in $\Phi^{c-\epsilon}$. Thus, ker $i_{*} \neq\{0\}$ (cf. $[7,13])$ and, by exactness, $H_{1}\left(\Phi^{c+\epsilon}, \Phi^{c-\epsilon}\right) \neq\{0\}$. It follows from theorem1.1 that

$$
\operatorname{dim} C_{1}(\Phi, u) \geq 1 .
$$

Now, we define the class of closed symmetric subsets of $\mathrm{X}$ as

$$
\Sigma=\{A \subset X: A \text { closed, } A=-A\} .
$$

Definition 1.3. For a non empty set $A$ in $\Sigma$, following Coffman (cf. [5]), we define the Krasnoselskii genus as

$$
\gamma(A)=\left\{\begin{array}{l}
\inf \left\{m: \exists h \in C\left(A, \mathbb{R}^{m} \backslash\{0\}\right) ; h(-x)=-h(x)\right\}, \\
\infty, \text { if }\{\ldots\} \text { is empty, in particular if } 0 \in A .
\end{array}\right.
$$

For A empty we define $\gamma(A)=0$.

We note

$$
A_{k}=\{C \in \Sigma: C \text { compact, } \gamma(C) \geq k\} .
$$

Our main tool for proving the existence of critical point for $\Phi$, will be the following minimax theorem due to the second author ( cf.Theorem 3.5, [10] ), with condition (C).

Theorem 1.3. Let $\Phi$ be a $C^{1}$ functional on $X$ satisfying $(C)$, let $Q$ be a closed connected subset of $X$ such that $\partial Q \cap \partial(-Q) \neq \emptyset$ and $\beta \in \mathbb{R}$. Assume that

(1) for every $K \in \mathcal{A}_{2}$, there exists $v_{K} \in K$ such that

$$
\Phi\left(v_{K}\right) \geq \beta \text { and } \Phi\left(-v_{K}\right) \geq \beta,
$$

(2) $a=\sup _{\partial Q} \Phi<\beta$

(3) $\sup \Phi<\infty$.

$$
\partial Q
$$

Then $\Phi$ has a critical value $c \geq \beta$ given by

$$
c=\inf _{h \in \Gamma} \sup _{x \in Q} \Phi(h(x)),
$$

where $\Gamma=\{h \in C(X, X): h(x)=x \quad$ for every $x \in \partial Q\}$.

\section{Proof of MAIN RESUlT}

In this section, we use theorem1.3 and we compute the critical group of $\Phi$ near zero. To do this, some technical lemmas are needed. First of all, we have to prove that the compactness condition (C) holds for $\Phi$.

Lemma 2.1. Assume $\left(f_{0}\right)$ and $\left(f_{1}\right)$. Then $\Phi$ satisfies the condition $(C)$. 
Proof. $c_{i}$ ) We first verify the Palais- Small condition is satisfied on the bounded subsets of $W_{0}^{1, p}(\Omega)$. Let $\left(u_{n}\right) \subset W_{0}^{1, p}(\Omega)$ be bounded such that

$$
\Phi^{\prime}\left(u_{n}\right) \rightarrow 0 \text { et } \Phi\left(u_{n}\right) \rightarrow c, c \in \mathbb{R}
$$

Passing if necessary to a subsequence, we may assume that

$$
\left\{\begin{array}{l}
u_{n} \rightarrow u \text { weakly in } W_{0}^{1, p}(\Omega), \\
u_{n} \rightarrow u \text { strongly in } L^{p}(\Omega), \\
u_{n}(x) \rightarrow u \text { a.e.in } \Omega .
\end{array}\right.
$$

From (2.1) and (2.2), we have

$$
\left\langle\Phi^{\prime}\left(u_{n}\right), u_{n}-u\right\rangle \rightarrow 0
$$

or equivalently

$$
\int_{\Omega}|\nabla u|^{p} \nabla u_{n} \nabla\left(u_{n}-u\right) d x-\int_{\Omega} f\left(x, u_{n}\right)\left(u_{n}-u\right) d x \rightarrow 0 .
$$

By the Hölder inequality, we obtain

$$
\int_{\Omega} f\left(x, u_{n}\right)\left(u_{n}-u\right) d x \rightarrow 0 .
$$

Thus, it follows from (2.3) and (2.4) that

$$
\left\langle-\Delta_{p} u_{n}, u_{n}-u\right\rangle \rightarrow 0 .
$$

Since $-\Delta_{p}$ is of type $S^{+}$(cf. [3]), we conclude that

$$
u_{n} \rightarrow u \text { strongly in } W_{0}^{1, p}(\Omega) .
$$

Let us now, by the contrary, show that $\left.c_{i i}\right)$ is satisfied for every $c \in \mathbb{R}$. Let $c \in \mathbb{R}$ and $\left(u_{n}\right) \subset W_{0}^{1, p}(\Omega)$ such that

Therefore

$$
\Phi\left(u_{n}\right) \rightarrow c, \quad\left\langle\Phi^{\prime}\left(u_{n}\right), u_{n}\right\rangle \rightarrow 0 \text { and }\left\|u_{n}\right\| \rightarrow+\infty
$$

$$
\lim _{n} \int_{\Omega}\left[p F\left(x, u_{n}\right)-u_{n} f\left(x, u_{n}\right)\right] d x=-p c .
$$

Taking $v_{n}=\frac{u_{n}}{\left\|u_{n}\right\|}$, we have $\left\|v_{n}\right\|=1$. Hence, without of loss generality, we may assume that there is $v \in W_{0}^{1, p}(\Omega)$ such that

$$
\left\{\begin{array}{l}
v_{n} \rightarrow v \text { weakly in } W_{0}^{1, p}(\Omega), \\
v_{n} \rightarrow v \text { strongly in } L^{p}(\Omega), \\
v_{n}(x) \rightarrow v(x) \text { a.e.in } \Omega .
\end{array}\right.
$$

On the other hand, in view $\left(f_{0}\right)$ and $\left(f_{1}\right)$, there exists $A>0$, and $B>0$ such that

$$
F(x, s) \leq A|s|^{p}+B, \quad \text { p.p } x \in \Omega .
$$

Via (2.5) and (2.8), we obtain

$$
\frac{1}{p}\left\|u_{n}\right\|^{p}-A\left\|u_{n}\right\|_{L^{p}}^{p}-B \leq C, \quad C \in \mathbb{R}
$$


After dividing by $\left\|u_{n}\right\|$ and passing to the limit, by using (2.7), we conclude

$$
\frac{1}{p}-A\|v\|_{L^{p}}^{p} \leq 0 \text { and } v \neq 0
$$

Let $\Omega_{0}=\{x \in \Omega: \quad v(x) \neq 0\}$, via the result above we have $\left|\Omega_{0}\right|>0$ and

$$
\left|u_{n}(x)\right| \rightarrow+\infty, \quad \text { p.p } x \in \Omega_{0}
$$

Furthermore, $\left(f_{0}\right)$ and $\left(f_{1}\right)$ implies that there exist $b(.) \in L^{1}(\Omega)$ such that

$$
p F(x, s)-s f(x, s) \geq b(x), \quad \text { p.p } x \in \Omega .
$$

Then

$$
\int_{\Omega}\left[p F\left(x, u_{n}\right)-u_{n} f\left(x, u_{n}\right)\right] d x \geq \int_{\Omega_{0}}\left[p F\left(x, u_{n}\right)-u_{n} f\left(x, u_{n}\right)\right]-\|b\|_{L^{1}} .
$$

However, (2.9) and Fatou's lemma 2.1 yields

$$
\lim _{n} \int_{\Omega}\left[p F\left(x, u_{n}\right)-u_{n} f\left(x, u_{n}\right)\right] d x=+\infty .
$$

This contradicts (2.6).

Now, we will show that the functional $\Phi$ satisfies the geometric conditions of Theorem 1.3. Let denote $E\left(\lambda_{1}\right)$ the eigenspace associated to the eigenvalue $\lambda_{1}$.

Lemma 2.2. assume $\left(f_{0}\right),\left(f_{1}\right)$ and $\left(f_{2}\right)$. Then

i) $\Phi(v) \rightarrow-\infty, \quad\|v\| \rightarrow+\infty, \quad v \in E\left(\lambda_{1}\right)$.

ii) $\forall K \in \mathcal{A}_{2}, \quad \exists v_{K} \in K, \quad \exists \beta \in \mathbb{R}: \quad \Phi\left(v_{K}\right) \geq \beta \quad$ et $\Phi\left(-v_{K}\right) \geq \beta$.

Proof. Putting

and

$$
g(x, s)=f(x, s)-\lambda_{1}|s|^{p-2} s,
$$

$$
G(x, s)=F(x, s)-\frac{\lambda_{1}}{P}|s|^{p} .
$$

i) Via $\left(f_{1}\right)$, for all $M>0$, there exist $s_{M} \geq 0$;

$$
s f(x, s)-p F(x, s) \leq-M, \text { pour }|s| \geq s_{M}, \quad \text { p.p } x \in \Omega .
$$

For $s \geq s_{M}$,

$$
\frac{d}{d s}\left[\frac{G(x, s)}{|s|^{p}}\right]=\frac{s g(x, s)-p G(x, s)}{s^{p+1}},
$$

by integrating over $[s, S] \subset\left[s_{M},+\infty[\right.$, it follows

$$
\frac{G(x, S)}{S^{p}}-\frac{G(x, s)}{s^{p}} \leq-\frac{M}{p}\left(\frac{1}{S^{p}}-\frac{1}{s^{p}}\right) .
$$

From $\left(f_{2}\right)$, we get

$$
\liminf _{S \rightarrow+\infty} \frac{G(x, S)}{S^{p}} \geq 0
$$

Thus,

$$
G(x, s) \geq \frac{M}{p}, \quad \forall s \geq s_{M}, \quad \text { p.p } x \in \Omega .
$$


Similar, we show that for every $s \leq-s_{M}, G(x, s) \geq \frac{M}{p}, \quad$ p.p $x \in \Omega$.

Since $\Phi(v)=-\int_{\Omega} G(x, v) d x$, for all $v \in E\left(\lambda_{1}\right)$ and $\lim _{|s| \rightarrow \infty} G(x, s)=+\infty$, the proof is completed .

ii) Let us recall that the Lusternik-Schnirelaman theory (cf. [1]) gives

$$
\lambda_{2}=\inf _{K \in \mathcal{A}_{2}} \sup \left\{\int|\nabla u|^{p} d x, \int|u|^{p} d x=1, u \in K\right\}
$$

Therefore,

$$
\forall K \in \mathcal{A}_{2}, \forall \varepsilon>0, \exists v_{K} \in K:\left(\lambda_{2}-\varepsilon\right) \int\left|v_{K}\right|^{p} d x \leq \int\left|\nabla v_{K}\right|^{p} d x .
$$

Indeed, if $0 \in K$, we take $v_{K}=0$.

Otherwise, we consider the odd mapping

$$
g: K \rightarrow K^{\prime}, v \mapsto \frac{v}{\|v\|_{L^{p}}} .
$$

By the genus properties we have $\gamma(g(K)) \geq 2$ and by the definition of $\lambda_{2}$ there exist $w_{K} \in K^{\prime}$ such that $\int\left|w_{K}\right|^{p} d x=1$ and

$$
\left(\lambda_{2}-\varepsilon\right) \leq \int\left|\nabla w_{K}\right|^{p} d x
$$

Thus (2.10) is satisfied by setting $v_{K}=g^{-1}\left(w_{K}\right)$.

On the other hand, $k(x)<\lambda_{2}$ and $\left(f_{0}\right)$ implies

$$
F(x, s) \leq\left(\lambda_{2}-2 \varepsilon\right) \frac{|s|^{p}}{p}+C, \quad \forall s \in \mathbb{R}, C>0 .
$$

Therefore, it follows from (2.10) and (2.11) that

$$
\begin{aligned}
\Phi\left(v_{K}\right) & \geq \frac{1}{p} \int\left|\nabla v_{K}\right|^{p} d x-\left(\frac{\lambda_{2}-2 \varepsilon}{p}\right) \int\left|v_{K}\right|^{p} d x-C|\Omega|, \\
& \geq \frac{1}{p}\left(1-\frac{\lambda_{2}-2 \varepsilon}{\lambda_{2}-\varepsilon}\right) \int\left|\nabla v_{K}\right|^{p} d x-C|\Omega| .
\end{aligned}
$$

The argument is similar for

$$
\Phi\left(-v_{K}\right) \geq \frac{1}{p}\left(1-\frac{\lambda_{2}-2 \varepsilon}{\lambda_{2}-\varepsilon}\right) \int\left|\nabla v_{K}\right|^{p} d x-C|\Omega| .
$$

So, for every $K \in \mathcal{A}_{2}$, we have

$$
\Phi\left( \pm v_{K}\right) \geq \beta:=-C|\Omega|
$$

We show now that the critical groups of $\Phi$ at zero are trivial. Note that the following lemma is proved in case $p=2$, see [14].

Lemma 2.3. Assume $\left(f_{0}\right),\left(f_{3}\right)$ and zero is an isolated critical point of $\Phi$. Then,

$$
C_{q}(\Phi, 0) \cong 0, \quad \forall q \in \mathbb{Z}
$$


Proof. The idea of its proof is similar to that of Theorem 1 in [14] and to one in [17]. Let denote by $B_{\rho}$ the closed ball in $W_{0}^{1, p}(\Omega)$ of radius $\rho>0$ with the center at the origin. The result will be proved by constructing a deformation mapping for the topological pairs $\left(B_{\rho}, B_{\rho} \backslash\{0\}\right)$ and $\left(B_{\rho} \cap \Phi^{0}, B_{\rho} \cap \Phi^{0} \backslash\{0\}\right)$. For this purpose, we need to analyze the local properties of $\Phi$ near zero. Thus, some technical affirmations must be proved.

It follows from $\left(f_{3}\right)$ that there exists a constant $c>0$ such that

$$
F(x, t) \geq c|t|^{\mu}, \quad \text { for } x \in \Omega, \quad|t| \leq \delta .
$$

Using $\left(f_{0}\right)$ and (2.14), we get

$$
F(x, t) \geq c|t|^{\mu}-c_{1}|t|^{q}, \quad x \in \Omega, \quad t \in \mathbb{R}
$$

for some $q \in\left(p, p^{*}\right)$ and $c_{1}>0$.

Then, for $u \neq 0$, zero is a local maximum for the functional $\Phi(t u), t \in \mathbb{R}$.

Indeed, for $u \in W_{0}^{1, p}(\Omega), u \neq 0$ and $s>0$, we have

$$
\begin{gathered}
\Phi(s u)=\frac{1}{p} s^{p} \int_{\Omega}|\nabla u|^{p} d x-\int_{\Omega} F(x, s u) d x \\
\leq \frac{s^{p}}{p}\|u\|^{p}-\int_{\Omega}\left(c|s u|^{\mu}-c_{1}|s u|^{q}\right) d x \\
\leq \frac{s^{p}}{p}\|u\|^{p}-c s^{\mu}\|u\|_{\mu}^{\mu}+c_{1} s^{q}\|u\|_{q}^{q} .
\end{gathered}
$$

Since $\mu<p<q$, there exists a $s_{0}=s_{0}(u)>0$ such that

Now, we affirm that there exists $\rho>0$ such that

$$
\Phi(s u)<0, \quad \text { for all } 0<s<s_{0} .
$$

$$
\left.\frac{d}{d s} \Phi(s u)\right|_{s=1}>0, \text { for all } u \in W_{0}^{1, p}(\Omega) \text { with } \Phi(u)=0 \text { and } 0<\|u\| \leq \rho
$$

In fact, for $u \in W_{0}^{1, p}(\Omega)$ be such that $\Phi(u)=0$. In return for $\left(f_{3}\right)$ and $\left(f_{0}\right)$ respectively, we have for $\varepsilon>0$ sufficiently small, there exists $r:=r(\varepsilon)>0$ such that

$$
\mu F(x, u)-f(x, u) u \geq(\alpha-\varepsilon)|u|^{p} \text {, a.e } x \in \Omega \text { and }|u| \leq r,
$$

and

$$
\mu F(x, u)-f(x, u) u \geq-c_{\varepsilon}|u|^{q}, \text { a.e } x \in \Omega \text { and }|u|>r,
$$

for some $q \in\left(p, p^{*}\right)$ and $c_{\varepsilon}>0$.

Denote by

$$
\Omega_{r}(u)=\{x \in \Omega:|u|>r\} \text { and } \Omega^{r}(u)=\{x \in \Omega:|u| \leq r\} .
$$

Then, since $\Phi(u)=0$ and by the Poincaré's inequality, we write

$$
\begin{aligned}
\left.\frac{d}{d s} \Phi(s u)\right|_{s=1} & =\left.\left\langle\Phi^{\prime}(s u), u\right\rangle\right|_{s=1} \\
& =\int_{\Omega}|\nabla u|^{p} d x-\int_{\Omega} f(x, u) u d x \\
& =\left(1-\frac{\mu}{p}\right) \int_{\Omega}|\nabla u|^{p} d x+\int_{\Omega^{r}(u)}(\mu F(x, u)-f(x, u) u) d x+\int_{\Omega_{r}(u)}(\mu F(x, u)-f(x, u) u) d x \\
\geq & \left(1-\frac{\mu}{p}\right)\|u\|^{p}+(\alpha-\varepsilon) \int_{\Omega^{r}(u)}|u|^{p} d x-c_{\varepsilon} \int_{\Omega_{r}(u)}|u|^{q} d x
\end{aligned}
$$




$$
\geq \theta\|u\|^{p}-C_{\varepsilon}\|u\|^{q}
$$

for some $C_{\varepsilon}>0$, where $\theta=\left(1-\frac{\mu}{p}+\frac{\alpha}{\lambda_{1}}-\frac{\varepsilon}{\lambda_{1}}\right)$.

Since $p<q,(2.18)$ is verified for $\varepsilon$ small enough such that $\theta>0$.

With the above affirmations, we are now able to prove the lemma 2.3. For a fixed $\rho>0$ such that zero is the unique critical point of $\Phi$ in $B_{\rho}$. We first claim that

$$
\Phi(s u) \leq 0, \text { for } s \in(0,1), \text { for all } u \in W_{0}^{1, p}(\Omega) \text { with } \Phi(u) \leq 0 \text { and }\|u\| \leq \rho
$$

Indeed, let $\|u\| \leq \rho$ with $\Phi(u) \leq 0$. By contradiction, assume that there is some $s_{0} \in(0,1]$ such that $\Phi\left(s_{0} u\right)>0$. Then, from the continuity of $\Phi$, there exists $s_{1} \in\left(s_{0}, 1\right]$ such that $\Phi\left(s_{1} u\right)=0$. Choose $s_{2} \in\left(s_{0}, 1\right]$ such that $s_{2}=\min \left\{s \in\left[s_{0}, 1\right]: \Phi(s u)=0\right\}$. It clear that $\Phi(s u) \geq 0$ for each $s \in\left[s_{0}, s_{2}\right]$. Putting $v=s_{2} u$, we have

$$
\Phi(s u)-\Phi\left(s_{2} u\right) \geq 0 \text { implies that }\left.\frac{d}{d s} \Phi(s u)\right|_{s=s_{2}}=\left.\frac{d}{d s} \Phi(s v)\right|_{s=1} \leq 0 .
$$

This is a contradiction with (2.18).

Define a mapping $h:[0,1] \times\left(B_{\rho} \cap \Phi^{0}\right) \rightarrow B_{\rho} \cap \Phi^{0}$, by

$$
h(s, u)=(1-s) u .
$$

Then $h$ is a deformation which deforms $B_{\rho} \cap \Phi^{0}$ into itself, that is, $B_{\rho} \cap \Phi^{0}$ is contractible in itself.

Now, we prove that $\left(B_{\rho} \cap \Phi^{0}\right) \backslash\{0\}$ is contractible in itself too. For this purpose, define a mapping $T$ : $B_{\rho} \backslash\{0\} \rightarrow(0,1]$ by

$$
\begin{gathered}
T(u)=1, \text { for } u \in\left(B_{\rho} \cap \Phi^{0}\right) \backslash\{0\}, \\
T(u)=s, \text { for } u \in B_{\rho} \backslash \Phi^{0} \text { with } \Phi(s u)=0, s<1 .
\end{gathered}
$$

From the relations $(2.17),(2.18)$ and $(2.19), T$ is well defined and if $\Phi(u)>0$ then, there exists an unique $T(u) \in(0,1)$ such that

$$
\left\{\begin{array}{l}
\Phi(s u)<0, \forall s \in(0, T(u)) \\
\Phi(T(u) u)=0 \\
\Phi(s u)>0, \forall s \in(T(u), 1)
\end{array}\right.
$$

Thus, using (2.18), (2.20) and the Implicit Function Theorem to get that the mapping $T$ is continuous.

Finally, we define a mapping $\eta:[0,1] \times B_{\rho} \rightarrow B_{\rho}$ by

$$
\eta(s, u)=h(s, u)+s T(u) u, \quad s \in[0,1], u \in B_{\rho} .
$$

The continuity of $h$ and $T$ implies that $\eta$ is continuous deformation from $\left(B_{\rho}, B_{\rho} \backslash\{0\}\right)$ to $\left(B_{\rho} \cap, \Phi^{0}, B_{\rho} \cap \Phi^{0} \backslash\{0\}\right)$. Since $W_{0}^{1, p}(\Omega)$ is infinite -dimensional, $B_{\rho} \backslash\{0\}$ is contractible in itself. So, by the fact that retracts of contractible space are also contractible, $B_{\rho} \cap \Phi^{0} \backslash\{0\}$ is contractible in itself.

Using the homotopy invariance of homology group, we get

$$
C_{q}(\Phi, 0)=H_{q}\left(B_{\rho} \cap \Phi^{0}, B_{\rho} \cap \Phi^{0} \backslash\{0\}\right) \cong H_{q}\left(B_{\rho}, B_{\rho} \backslash\{0\}\right), \quad \forall q \in \mathbb{Z}
$$

Since $B_{\rho} \backslash\{0\}$ is contractible, we have

$$
H_{q}\left(B_{\rho}, B_{\rho} \backslash\{0\}\right) \cong 0, \quad \forall q \in \mathbb{Z}
$$

The proof of lemma 2.3 is completed. 


\section{Proof of main result}

In view of lemmas 2.1 and 2.2 , we may apply Theorem 1.3. Letting $Q=\left\{t \varphi_{1}:|t| \leq R\right\}, R>0$. Obviously, $\mathrm{Q}$ is closed and compact. Also, by lemma 2.2, for some large enough $\mathrm{R}$, we have

$$
\sup _{\partial \Omega} \Phi<\beta
$$

Therefore, the functional $\Phi$ has a critical value

$$
c=\inf _{h \in \Gamma} \sup _{x \in Q} \Phi(h(x)) \geq \beta
$$

where $\Gamma=\left\{h \in C\left(W_{0}^{1, p}(\Omega), W_{0}^{1, p}(\Omega)\right): h(x)=x \quad\right.$ for every $\left.x \in\left\{-R \varphi_{1}, R \varphi_{1}\right\}\right\}$.

So, there exists at least one critical point $u^{*}$ of $\Phi$. More precisely, $u^{*}$ is a Mountain Pass point. Then, by theorem 1.2 , we have $C_{1}\left(\Phi, u^{*}\right) \not 0$. However $C_{1}(\Phi, 0) \cong 0$ by lemma 2.3 . Hence, $u^{*} \neq 0$.

\section{REFERENCES}

[1] H.Amann, Lusternik-Schnirelman theory and non-linear eigenvalue problems, Math.Ann. 199 (1972),55-72.

[2] A.Anane And N.Tsouli, On the second eigenvalue of the p-Laplacien, Nonlinear Partiel Differential Equations, Pitmen research Notes 343(1996), 1-9.

[3] J.Berkovits and V.Mustonen, Nonlinear mappings of monotone type(classification and degree theory), Math. Univer. Oulu, Linnanmaa, Finland, 1988

[4] G.Cerami, Un criterio di esistenza per i punti citici su varieta'illimitate, Rend. Acad. Sci. Lett. Ist. Lambardo, 112(1978), $332-336$.

[5] C.V.Coffman, A minimum-maximum principle for a class of nonlinear integral equations, J. Analyse Math. 22(1969), 14011412.

[6] D.G.Costa and C.A.Magalhães, Existence results for perturbations of the p-Laplacian, Non. Anal. T. M. A 24(1995), 409-418.

[7] K.Chang, Infinite Dimensional Morse theory and Multiple Solution Problems, Birkhäuser, Boston, 1993.

[8] A.R.El Amrouss, Théorèmes de points critiques et applications aux problèmes variationnels non linéaires, Thèse de Doctorat, Université Mohammed I Oujda, 1998.

[9] A.R.El Amrouss et M.Moussaoui, Minimax principle for critical point theory in applications to quasilinear boundary value problems, Elec.J.Diff. Equa, 18(2000), 1-9.

[10] A.R.El Amrouss, Critical Point Theorems and Applications to Differential Equations, Acta Mathematica Sinica, English Series, Vol.21, No.1 (2005), 129-142.

[11] J.Liu et J.Su, Remarks on Multiple Nontrivial Solutions for Quasi-Lineat Resonant Problems, Journal of Mathematical Analysis and Applications ,258(2001), 209-222.

[12] E.N.Dancer And K.Perera, Some remarks on the Fucik spectrum of the p-Laplacian and critical groups, J. Math. Anal. Appl.254(2001), 164-177.

[13] J.Mawhin and M.Willem, Critical Point Theory and Hamiltonien systems, Springer-Verlag, New-York, 1989.

[14] V.Monoz, Solutions of superlinear at zero elliptic equations via Morse Theory, Topol. Methods in Nonlinear Anal.10(1997),387397.

[15] Shibo Liu, Existence of solutions to a superlinear p-Laplacien equation.Elec. J. Diff. Equa, 66(2001), 1-6.

[16] E.A.B.Silva, Critical point theorems and applications to semilnear elliptic problem, NoDEA 3(1996), 245-261.

[17] Z.Q.WANG, On a superlinear elliptic equation, Ann. Inst. Henré Poincaré, Analyse non linéaire, 8(1991), 43-51. 